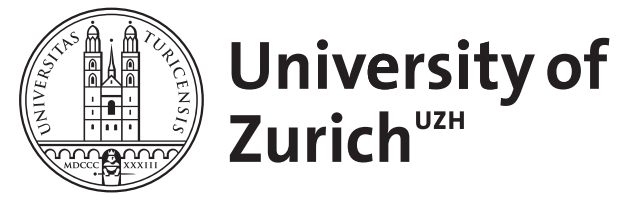

\title{
Blut ab ano
}

Haller, S ; Krayenbühl, P A ; Gubler, C

DOI: https://doi.org/10.1024/1661-8157/a000806

Posted at the Zurich Open Repository and Archive, University of Zurich ZORA URL: https://doi.org/10.5167/uzh-67472

Journal Article

Accepted Version

Originally published at:

Haller, S; Krayenbühl, P A; Gubler, C (2012). Blut ab ano. Praxis, 101(1):3-13.

DOI: https://doi.org/10.1024/1661-8157/a000806 
DOI 10.1024/1661-8157/a000806

((CME))

\section{Blut ab ano}

\section{Einführung}

Blutverlust aus dem Gastrointestinaltrakt ist ein häufiges Problem in Notfallstationen, die Gesamtmortalität in der Praxis beträgt ungefähr 10\% (obere intestinale Blutungen) bzw. 5\% (untere intestinale Blutungen) [2,3]. Insgesamt sind von gastrointestinalen Blutungen (GIB) häufiger Männer und ältere Patienten betroffen [3,4]. Das Risiko für eine Blutung steigt mit dem Lebensalter; das Durchschnittsalter für das Auftreten einer unteren GIB beträgt 70 Jahre. Zu den häufigsten zugrundeliegenden Ursachen von GIB gehören Karzinome, Ulzerationen, portale Hypertonie, vaskuläre Malformationen und Entzündungen. Zusätzlich müssen die verbreitete Verwendung von NSAR, Antikoagulanzien und Plättchenhemmern beim Management von Patienten mit Blutungen beachtet werden. Der zunehmende Gebrauch dieser Medikamente, insbesondere in Kombination, und die damit verbundene erhöhte Blutungsgefahr dürften auch massgeblich daran beteiligt sein, dass sich die Gesamtmortalität über die letzen 30 Jahre kaum verändert hat [5]. Die Herangehensweise an den Patienten wird durch das Ausmass der Blutung, der anatomischen Zuordnung des Blutverlusts und der zugrundeliegenden Pathophysiologie mit allfälligen Risikofaktoren bestimmt. Neue diagnostische und therapeutische Massnahmen haben die Behandlungsoptionen erweitert. Dabei erfordert die Therapie oft einen multidisziplinären Ansatz.

\section{Im Artikel verwendete Abkürzungen}

GIB Gastrointestinal-Blutung

NSAR Nicht-steroidale Antirheumatika

CT Computer-Tomographie

MRI Magnetresonanz-Tomographie 
Definition / Einteilung / Ursachen / differentialdiagnostische Überlegungen

\section{Einteilung und Definition}

Bei GIB wird zwischen akuten und chronischen Blutungen unterschieden. Die akute GIB ist definiert als eine weniger als drei Tage andauernde Blutung, wohingegen es sich bei der chronischen Blutung um einen intermittierenden und/oder langsamen Blutverlust handelt.

Weiterhin wird unterteilt in overte und okkulte GIB. Eine overte GIB ist eine klinisch evidente Blutung mit sichtbarem Blut. Der Begriff okkulte Blutung bezieht sich auf eine Präsentation der GIB durch einen positiven Fäkaltest und/oder durch eine Eisenmangelanämie, ohne klinisch sichtbaren Blutverlust.

Sowohl die overte als auch die okkulte GIB können sich als obskure Blutung präsentieren. Bei der obskuren Blutung handelt es sich um eine Blutung aus dem Gastrointestinaltrakt, die anhaltend oder wiederholt auftritt, ohne eine identifizierte Blutungsquelle nach oberer und unterer Endoskopie und Abklärung des Dünndarms aufzuweisen.

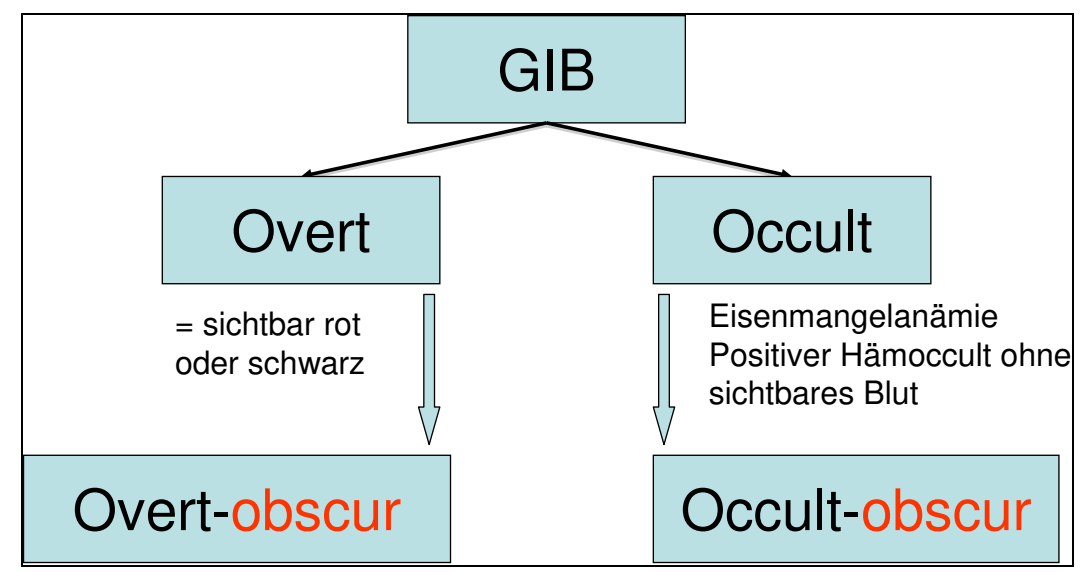

((Layout: Occult als okkult schreiben, Positiver als positiver, Overt als overt, obskur als obskur,))

Abb. 1: Einteilung der Gastrointestinal-Blutungen. (Obskur=Ursache unklar nach Gastroskopie, Koloskopie und Dünndarmabklärung)

Obskure Blutungen treten bei ca. 5\% der GIB auf. Bei etwa 75\% der obskuren Blutungen befindet sich die Blutungsquelle im Dünndarm.

Bei der Blutungsquelle wird zwischen oberen und unteren intestinalen Blutungen unterschieden, entsprechend der Lokalisation proximal oder distal des Treitz'schen Ligaments.

\section{Blutungsursachen und Differentialdiagnosen}

Zu den häufigsten oberen GIB gehört das peptische Ulkus, das 30-50\% aller Fälle von oberen GIB ausmacht und oft als Folge einer Helicobacter-pylori-Infektion oder von NSAR-Gebrauch auftritt. Weitere häufige Ursachen sind die Ösophagusvarizen-Blutung, Erosionen, Mallory-Weiss-Läsionen und Neoplasien. 


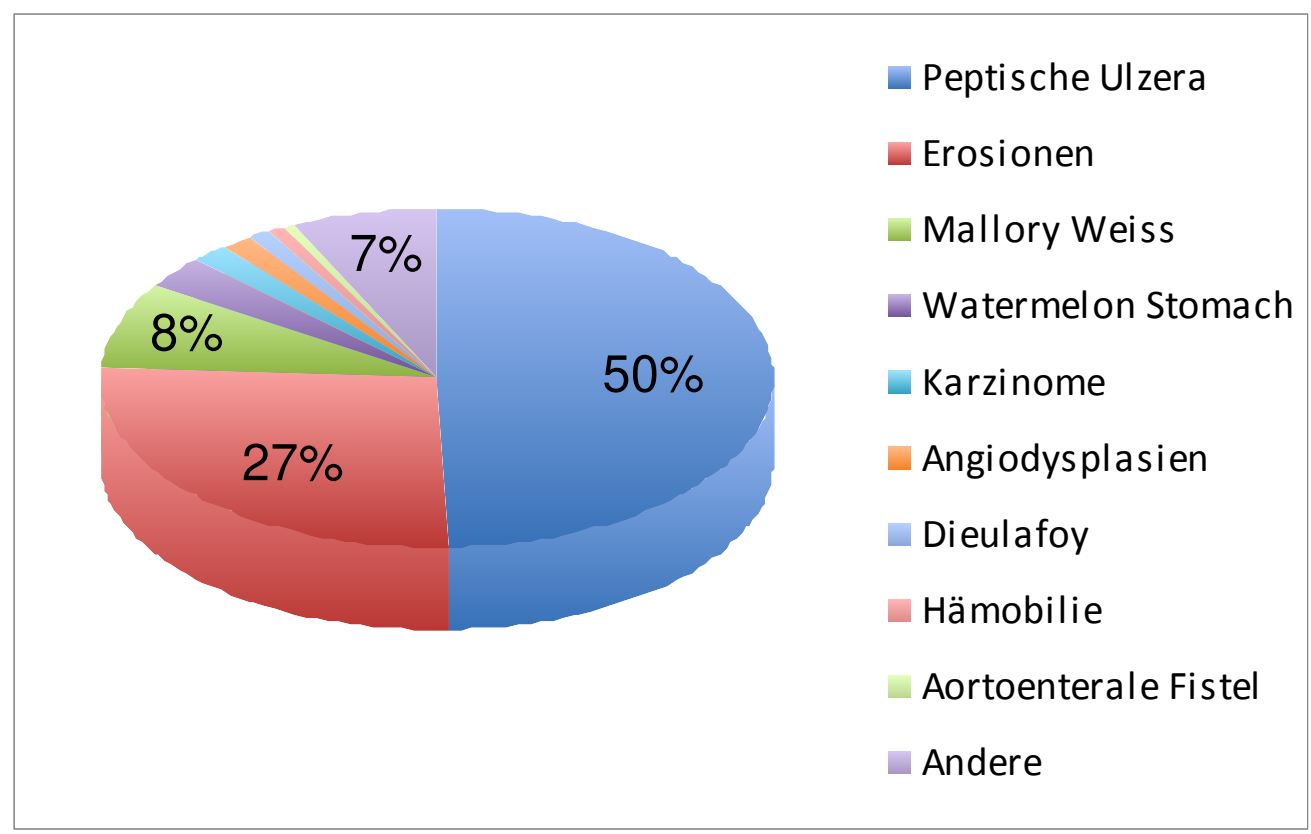

Abb. 2: Häufigkeit nach Ätiologie oberer Gastrointestinalblutungen [12,13].

Bei der unteren GIB findet sich die Blutungsquelle meist im Kolon. Die häufigsten Ursachen sind Divertikelblutungen, Angiodysplasien, ischämische Kolitiden, Colitis ulcerosa und die Hämorrhoidalblutung (Tab. 1). In bis zu $20 \%$ der Fälle werden obere GIB und Dünndarmblutungen initial als untere GIB missinterpretiert.

Tab. 1: Häufigste Ursachen von unteren Gastrointestinal-Blutungen [14,15

\begin{tabular}{|l|l|}
\hline Ursachen & Prävalenz \\
\hline Divertikel-Blutung & $17-40 \%$ \\
\hline Angiodysplasie & $9-21 \%$ \\
\hline $\begin{array}{l}\text { Kolitis (ischämisch, infektiös, chronisch entzündliche Darmerkrankung, } \\
\text { Strahlenkolitis) }\end{array}$ & $2-30 \%$ \\
\hline $\begin{array}{l}\text { Post-Polypektomie-Blutung } \\
\text { (Hämorrhoiden, Rektalvarizen, Fissuren) }\end{array}$ & $11-14 \%$ \\
\hline Dünndarmblutung & $4-10 \%$ \\
\hline Obere Gastrointestinal-Blutung & $2-9 \%$ \\
\hline
\end{tabular}

Divertikelblutungen werden in 17-40\% der Fälle von unteren GIB diagnostiziert. Sie treten bei bis zu 15\% der Patienten mit einer Divertikulose auf, eine Komplikation, die insbesondere bei älteren Personen auftritt. Während eine Divertikulose in der westlichen Welt häufiger im Sigma oder linken Kolon vorkommt, treten Divertikelblutungen typischerweise im rechten Kolon auf. Als zusätzlicher Risikofaktor gilt die NSAR-Einnahme, differentialdiagnostisch kommt die zu wenig bekannte NSAR-assoziierte Kolonulzeration in Frage [6]. Üblicherweise bestehen keine zusätzlichen Symptome, der Blutabgang ist schmerzlos. Eine Divertikulitis und eine Blutung koexistieren so gut wie nie. 
Ischämische Kolitiden, welche für bis zu 19\% der unteren GIB verantwortlich sind, entstehen durch eine plötzliche temporäre Reduktion der Mesenterialdurchblutung durch Hypotonie, Embolisation (Vorhofflimmern) oder Vasospasmen. Eine solche Hypoperfusion betrifft typischerweise die «Wasserscheiden»-Bereiche des Kolons, namentlich die splenische Flexur, und den rektosigmoidalen Übergang. Es wird postuliert, dass die Inzidenz der ischämischen Kolitiden ansteigt aufgrund der zunehmenden Anzahl älterer Patienten mit kardiovaskulären Erkrankungen.

Angiodysplasien machen bis zu 21\% der unteren GIB aus, es handelt sich um dünnkalibrige, dilatierte, (sub)muköse Gefässe. Betroffene Patienten präsentieren sich mit okkulter Blutung oder aber mit Frischblut ab ano, die von einer Divertikelblutung nicht zu unterscheiden ist.

Typische Ursachen für kleine Mengen Frischblut im oder meist auf dem Stuhl sind Hämorrhoiden (schmerzlos), Analfissuren (schmerzhaft), Polypen, Proctitis, rektale Ulzera und Karzinome.

Weitere Ursachen für untere GIB sind chronisch entzündliche Darmerkrankungen, Neoplasien, infektiöse Kolitiden, Strahlenkolitiden (meistens im Rektum), Meckel-Divertikel, post-Polypektomie-Blutungen und selten Kolonvarizen. Selten kann die Blutungsursache auch ausserhalb des Intestinallumens liegen, wie es bei der Hämobilie oder dem Hämosuccus pancreaticus der Fall ist. Die relative Häufigkeit dieser Blutungsursachen hängt unter anderem vom Alter der Patienten ab (Tab. 2).

Tab. 2: Altersabhängige Differentialdiagnosen der obskuren Gastrointestinalblutungen (adaptiert nach [16]).

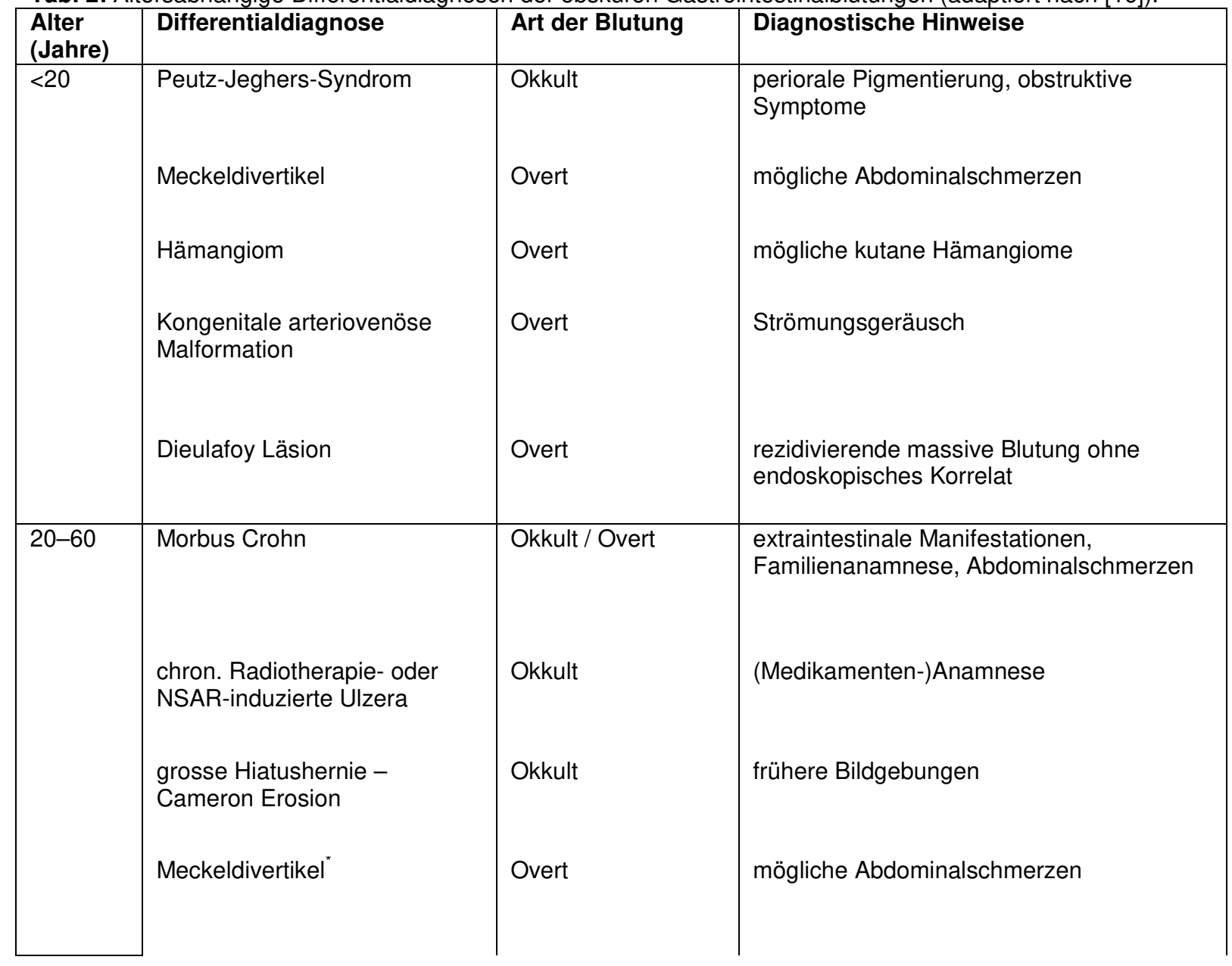




\begin{tabular}{|c|c|c|c|}
\hline & $\begin{array}{l}\text { vaskuläre Ektasie des } \\
\text { Magenantrums (watermelon } \\
\text { stomach) } \\
\text { Post-Radiatio-Angioektasie } \\
\text { Dieulafoy Läsion } \\
\text { Tumore } \\
\text { Von Willebrand-Erkrankung }\end{array}$ & $\begin{array}{l}\text { Okkult/Overt } \\
\text { Okkult/Overt } \\
\text { Overt } \\
\text { Okkult/Overt } \\
\text { Okkult }\end{array}$ & $\begin{array}{l}\text { Anamnese } \\
\text { rezidivierende massive Blutung ohne } \\
\text { endoskopisches Korrelat } \\
\text { Gewichtsverlust, Lymphadenopathie } \\
\text { Epistaxis; andere Blutungen }\end{array}$ \\
\hline$>60$ & $\begin{array}{l}\text { Amyloidose } \\
\text { Angiektasie } \\
\text { Hereditäre hämorrhagische } \\
\text { Teleangiektasie } \\
\text { Malignome } \\
\end{array}$ & $\begin{array}{l}\text { Okkult / Overt } \\
\text { Okkult / Overt } \\
\text { Okkult / Overt } \\
\text { Okkult / Overt }\end{array}$ & $\begin{array}{l}\text { Organomegalie, Purpura, renale } \\
\text { Beteiligung } \\
\text { Teleangiektasien an Mund, Gesicht, } \\
\text { Fingern } \\
\text { B-Symptomatik, Schmerzen }\end{array}$ \\
\hline
\end{tabular}

* bis Alter 35-40 Jahre

B-Symptomatik, Schmerzen

** beinhaltet alle Diagnosen der Altersgruppe 20-60 Jahre 


\section{g \\ C. Klinik / Evaluation}

\section{Evaluation - Anamnese und Klinik}

Patienten mit unterer GIB präsentieren sich mit Hämatochezie (helles rotes oder rotbraunes Blut ab ano), Blutkoagel ab ano oder Meläna (Teerstuhl), bei oberen GIB kann zudem Hämatemesis (Bluterbrechen) auftreten. Gerade bei kleinen Mengen Blut im Stuhl ist das Ziel der klinischen Evaluation, jene Patienten zu identifizieren, die ein ausreichend hohes Risiko für eine ernsthafte Blutungsursache haben und einer weiteren Abklärung bedürfen.

Anamnestisch kleine Mengen roten Blutes im oder auf dem Stuhl suggerieren eine Blutungsquelle in der Nähe des Analkanals. Eine Auflagerung auf dem Stuhl kann auf den Analkanal selbst (Hämorrhoiden, Fissur) als Quelle hinweisen. Dabei gilt es zu bedenken, dass die Wahrnehmung der Stuhlfarbe von Patienten und Ärzten sehr unterschiedlich ausfallen kann. Die Annahme einer anorektalen Blutungsquelle wird unterstützt durch anamnestische Angaben von wenig Blut am Toilettenpapier und Tropfen von Blut in die Toilette nach Defäkation.

Das Auftreten einer Meläna spricht eher für eine Blutungsquelle im oberen Gastrointestinal-Trakt und kann schon bei 150-200 ml Blutverlust auftreten. Eine Meläna kann jedoch von jeglicher Blutunglokalisation von der Nasenschleimhaut bis zum linken Kolon stammen. Hämatochezie infolge einer oberen GIB legt hingegen einen raschen Blutverlust von mindestens $1000 \mathrm{ml}$ nahe und kommt nur bei Varizenblutungen oder arteriell spritzender Ulkusblutung im Magen oder Duodenum vor. Ungefähr 10-15\% aller Patienten mit Hämatochezie haben eine obere gastrointestinale Blutungsquelle.

Bei Patienten mit vermuteter unterer GIB sollten anamnestisch frühere Blutungsepisoden und der Gebrauch von Antikoagulanzien, Plättchenhemmern, Aspirin und NSAR erfragt werden. Hierbei gilt es auch die Einnahme von frei verkäuflichen Medikamenten zu erfahren, die NSAR enthalten können. Die persönliche Anamnese, insbesondere hinsichtlich Koagulopathien, chronisch-entzündlichen Darmerkrankungen sowie Radiotherapien des Beckens für Prostata-, Rektal- oder Zervikalkarzinom spielen eine wichtige Rolle. Zudem sollten die Befunde früherer endoskopischer Untersuchungen eingeholt werden, insbesondere bezüglich Polypektomien in den vorangegangenen zwei Wochen.

Bei vermuteter Quelle der Blutung im oberen Gastrointestinaltrakt sollte neben der Einnahme von NSAR und Antikoagulanzien auch ein besonderes Augenmerk auf chronische Leber- und Ulkuserkrankungen gerichtet werden.

Die Familienanamnese bezüglich Kolonkarzinom, Polypen-Syndromen und die Anamnese des Alkohol- und Zigarettenkonsums helfen bei der Risikostratifizierung hinsichtlich gastrointestinaler Malignität weiter. Die Anamnese einer chronisch-entzündlichen Darmerkrankung ist wichtig, da bereits nach acht Jahren Krankheitsdauer das Risiko eines Kolonkarzinoms ansteigt.

\section{Begleit- und Warnsymptome}

Als assoziierte Symptome einer GIB können arterielle Hypotonie, Orthostase, Synkope, Thoraxschmerzen und Dyspnoe auftreten. Diese Befunde weisen auf einen schnellen gastrointestinalen Blutverlust hin. Eine Ruhetachykardie (Frequenz >100/min) zeigt einen akuten Blutverlust von 15-30\% des Blutvolumens an, wohingegen ein hypotoner Blutdruck mit einem Verlust von über 30\% einhergeht. Symptome eines schwerwiegenden Blutverlusts sind Tachypnoe und Veränderungen des Mentalstatus. 
Begleitende systemische Symptome wie Nachtschweiss, Fieber oder Gewichtsverlust legen ein malignes Geschehen oder eine chronische Infektion nahe. Das Alter ist ein bedeutender Risikofaktor für ein kolorektales Karzinom. Es ist eine seltene Diagnose vor dem 40. Lebensjahr, die Inzidenz beginnt jedoch zwischen dem Alter von 40 bis 50 Jahren signifikant zu steigen und nimmt in jeder nachfolgenden Altersdekade weiter zu [7]. 


\section{Klinisches Management}

Die initiale Beurteilung eines Patienten mit einer akuten GIB sollte sich primär auf Folgendes konzentrieren: Prüfung der Vitalzeichen, Erhebung einer gründlichen aber fokussierten Anamnese, Durchführung einer körperlichen Untersuchung mit Hauptaugenmerk auf die hämodynamische Situation und relevante Blutungszeichen. Die klinische Untersuchung sollte immer eine Examination des Anus und eine digitale rektale Untersuchung einschliessen. Zu den ersten Schritten gehört auch die Abnahme der wichtigsten Laborparameter (Hämoglobin, Hämatokrit, Harnstoff und Kreatinin, Gerinnungsprofil, Thrombozytenzahl und

Leberfunktionsdiagnostik) inklusive Testblut. Dabei muss bedacht werden, dass Hämoglobin und Hämatokrit in den ersten 24 Stunden aufgrund der Hämokonzentration oft unzuverlässige Parameter für den Blutverlust sind. Bei einer schweren Blutung steht die Volumengabe im Vordergrund, endoskopische Abklärungen sollten generell nur unternommen werden, wenn der Patient hämodynamisch stabil ist. Zur Volumensubstitution sollten zwei grosskalibrige periphere Zugänge zur Verfügung stehen. Eine signifikante GIB kann zu einem vermindertem Sauerstoffangebot am Herz und somit zu einer myokardialen Ischämie und Infarzierung führen, dies gilt es insbesondere bei Patienten mit bekannter koronarer Herzkrankheit zu beachten.

Bei oberen GIB reduziert die Gabe von intravenösen Protonenpumpenhemmern das Risiko einer Rezidivblutung. Falls eine Varizenblutung nicht ausgeschlossen werden kann, sollen bereits vor der Endoskopie zusätzlich Vasoaktiva zur mesenterialen Vasodilatation (Sandostatin, Vasopressin oder Terlipressin) verabreicht werden. Bei allen Patienten sollten NSAR und wenn aufgrund der Komorbiditäten vertretbar, die Antikogulanzien und Plättchenhemmer sistiert werden. In Fällen von milder Blutung kann dies sogar ausreichen, um die GIB zu beheben. Bei Patienten mit drug eluting stents ist der zeitliche Abstand zur Implantation der Stents relevant und das Absetzen, bzw. das Wiedereinsetzen der Medikation soll mit dem betreuenden Kardiologen koordiniert werden. 
Abklärungsstrategie / Diagnostik / Therapie / Komplikationen

Eine Gastroduodenoskopie sollte bei einer akuten oberen GIB innerhalb der ersten 12-24 Stunden durchgeführt werden. Bei Patienten mit raschem Blutverlust und Kreislaufkompromittierung oder mit vermuteter/bekannter schwerer Lebererkrankung soll die Endoskopie notfallmässig erfolgen. Eine obere Endoskopie kann die Blutungsquelle ermitteln und Hochrisikoläsionen, (z.B. blutendes Ulkus, Ösophagusvarizen) von Niedrigrisikoläsionen, (z.B. Mallory-Weiss-Läsion) unterscheiden und falls nötig direkt therapieren. Die Indikation zur Intubation ist bei instabilen Patienten grosszügig zu stellen. Bei stabilen Patienten ist die Regel betreffend Nüchternheit (sechs Stunden nach fester Nahrung, zwei Stunden nach Flüssigkeiten) einzuhalten. Patienten mit einer Meläna, dunkelrotem Blut ab ano oder orthostatischer Hypotonie sollten als Erstes eine Gastroskopie erhalten, da eine höhere Wahrscheinlichkeit einer oberen GIB vorliegt.

Bei vermuteter akuter unterer GIB wird innerhalb der ersten 24 bis 48 Stunden eine Koloskopie empfohlen. Eine frühe Koloskopie ist mit einer verminderten Hospitalisationsdauer assoziiert. Obwohl die meisten unteren GIB spontan sistieren, ist es wichtig die Ursache der Blutung zu ermitteln oder Differenzialdiagnosen auszuschliessen.

Die flexible Sigmoidoskopie untersucht die distalen $60 \mathrm{~cm}$ des Kolons. Als Vorteil gegenüber einer Koloskopie ist bei der Sigmoidoskopie eine Darmvorbereitung und Sedation nicht notwendig. Der Hauptnachteil besteht in der Reichweite, so dass nachfolgend oft eine Koloskopie notwendig wird, um weiter proximale Läsionen zu suchen. Läsionen im Magen- oder Darmlumen können endoskopisch bei bis zu 10\% verpasst werden, dies insbesondere in Notfallsituationen oder bei unzureichend vorbereitetem Kolon. Es ist daher bei obskurer Blutung angezeigt, die obere und/oder untere Endoskopie mindestens einmal zu wiederholen.

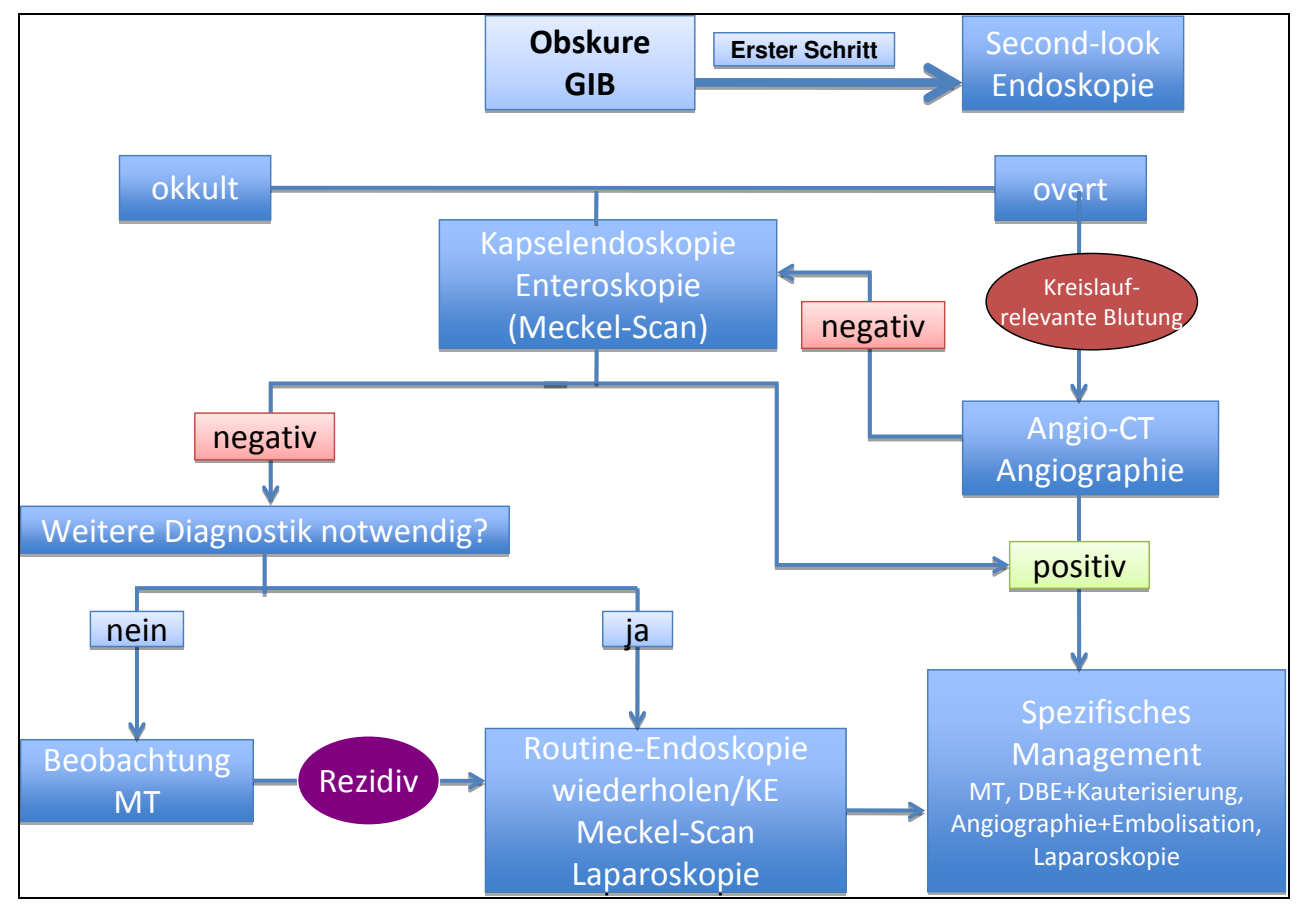

((Layout: Kasten rechtes, 2.Ebene: vertikale Linie zu lang))

((Autor: Vertikale Linie zwischen 1. und 2. Ebene fehlt, bitte angeben)) 
Abb. 3: Diagnostik und Management obskurer gastrointestinaler Blutungen (adaptiert nach [11]).

KE: Kapselendoskopie; DBE: Doppelballon-Enteroskopie; MT: Medikamentöse Therapie

Akut blutende Nicht-Varizen-Läsionen können endoskopisch mittels Unterspritzung von Adrenalin,

Thermokoagulation, Clipapplikation oder Fibrinkleber behandelt werden. Oft werden die Modalitäten kombiniert.

Die Therapie der Ösophagusvarizen erfolgt mittels mehrfacher endoskopischer Gummibandligatur oder Histoacrylinjektion beim Vorliegen von Fundusvarizen.

Bei nicht-stillbaren GIB als Folge einer portalen Hypertonie oder Varizen an atypischen Lokalisationen wie Kolon und Rektum kann ein durch interventionelle Radiologen angelegter Notfall-TIPSS (Trans-Jugulärer PortoSystemischer Shunt) die letzte und beste Option sein. Eine Folge des funktionierenden Shuntes kann eine hepatische Enzephalopathie sein.

Zur Evaluation des Dünndarms kann eine Kapselendoskopie durchgeführt werden. Hierfür wird eine Videokapsel geschluckt, die durch die intestinale Motilität durch den Magen in den Dünndarm transportiert wird. Die häufigste Komplikation ist eine Retention der Kapsel durch eine obstruierende Masse oder Striktur.

Die Kapselendoskopie hat ihre Stärken in der Beurteilung der Mukosa (Ulzera, Crohn, Angiodysplasien). Luminale Tumoren oder Divertikel im Dünndarm dagegen werden besser mittels selektiver Dünndarmdarstellung im MRI oder CT dargestellt.

Die Enteroskopie verbindet Diagnostik und allenfalls Therapie, ist jedoch immer aufwändig und nicht notfallmässig verfügbar. Zur Zeit ist die Enteroskopie in Singleballon-, Doppelballon- oder Spiral-overtube-Technik verfügbar. Alle Methoden können von oral oder anal angewandt werden, je nach vermuteter Lokalisation im Dünndarm. Als Entscheidungshilfe hierfür dient die Anamnese oder eine vorgängige Kapselendoskopie. Die Hauptkomplikation dieser Eingriffe ist eine Darmperforation.

Wird endoskopisch keine Blutungsquelle gefunden, kann entweder eine Angiographie oder eine Blutungsszintiggraphie durchgeführt werden. Die Voraussetzung für eine erfolgreiche Suche mittels

Angiographie ist ein Blutverlust von mindestens $1 \mathrm{ml} / \mathrm{min}$, das heisst drei bis vier Erythrozytenkonzentrate pro 24 Stunden. Im Falle einer nachweisbaren Blutungsquelle kann mittels Angiographie in 70-90\% der Fälle eine Hämostase durch Embolisation erreicht werden. Komplikationen treten in ca. 11\% der therapeutischen Embolisationen auf.

In der Tc-99m-Pertechnetat-Szintigraphie kann eine Blutung bei viel niedrigerer Blutungsrate (ab $0.1 \mathrm{ml} / \mathrm{min}$ ) identifiziert werden als in der Angiographie. Die Befunde einer Szintigraphie sind allerdings unspezifisch, eine Blutung kann einer generellen Region zugeordnet werden, die Zuordnung zu Kolon oder Dünndarm ist schwierig. Bei hoher Vortestwahrschinlichkeit für ein Meckeldivertikel (jüngere Patienten mit intermitterender Hämatochezie) soll eine Tc-99m-Pertechnetat-Szintigraphie verordnet werden.

Bei einer massiven oder schockierenden unteren GIB ist keine Endoskopie indiziert, sondern eine notfallmässige Angio-Computertomographie; je nach Befund wird direkt oder kombiniert konventionell angiographiert.

Falls eine Blutung persistiert, ist eine chirurgische Therapie zu erwägen. Dies bedingt jedoch eine grobe anatomische Zuordnung, um den Zugang primär richtig wählen zu können: Die Entscheidung Gastrotomie oder Duodenotomie versus Kolon- oder Dünndarmresektion ist präoperativ zu fällen. Bei Dünndarmblutungen ist eine intraoperative Endoskopie von grossem Nutzen. Ein chirurgisches Vorgehen bei Patienten mit aktiver Blutung ist mit signifikant höherer Morbidität und Mortalität vergesellschaftet. 


\section{Fallbericht Blut ab ano}

Y

Anamnese der Patientin

\section{Weg zur Diagnose/Kommentar}

Notfallmässige Selbstvorstellung der 47-jährigen, thailändischen Patientin am frühen Morgen mit kurzeitigen, passageren, abdominalen Schmerzen am Vorabend und mehrfachem, breiigem, dunklem bis schwarzem Stuhlgang über Nacht. Zudem leichter Schwindel. Keine Thoraxschmerzen.

Die Patientin konsumiert weder Alkohol noch Zigaretten. Es besteht kein Gebrauch von Aspirin, NSAR oder freiverkäuflichen Schmerzmedikamenten. Das Gewicht ist stabil, keine B-Symptomatik.

\section{Persönliche Anamnese:}

2003 Echinokokkeninfektion mit Leberteilresektion, Zwerchfellteilresektion, Y-Roux-Hepatojejunostomie (in Thailand 2003).

Portale Hypertonie bei chronischem Verschluss der V. portae.

2007 Histoacrylsklerosierung Fundusvarizen. 09/2010 Oesophagogastroduodenoskopie: Kleine Ösophagusvarizen, keine Fundusvarizen.
Dunkler bis schwarzer Stuhl spricht für eine Meläna.

Schwindel kann auf erhöhten Blutverlust hinweisen.

Keine Einnahme von

Thrombozytenaggregations-Hemmern.

Voroperation im Bereich des Dünndarms.

Portale Hypertonie, St.n. Fundusvarizen. Leberteilresektion.

Fundusvarizen vorbekannt.

\section{$d$}

Befunde der Patientin

\section{Weg zur Diagnose/Kommentar}

\section{Status}

48-jährige Patientin in reduziertem $A Z$, schlankem $E Z$ $(147 \mathrm{~cm}, 41 \mathrm{~kg})$. Temperatur aurikulär $37.7^{\circ} \mathrm{C}$. BD 105/71 mmHg, Puls 83/min regelmässig. Abdomen weich, Druckdolenz epigastrisch, kein Peritonismus, lebhafte Darmgeräusche über allen Quadranten, Leber einen Querfinger unter Rippenbogen palpabel, Milz nicht palpabel, reizlose quere Laparotomienarbe. Rektal glatte Schleimhaut, keine Raumforderung, normaler Sphinktertonus, wenig dunkles Blut am

Die Patientin ist hämodynamisch stabil. Es liegt somit aktuell kein volumenrelevanter Blutverlust vor.

Blutnachweis rektal. 


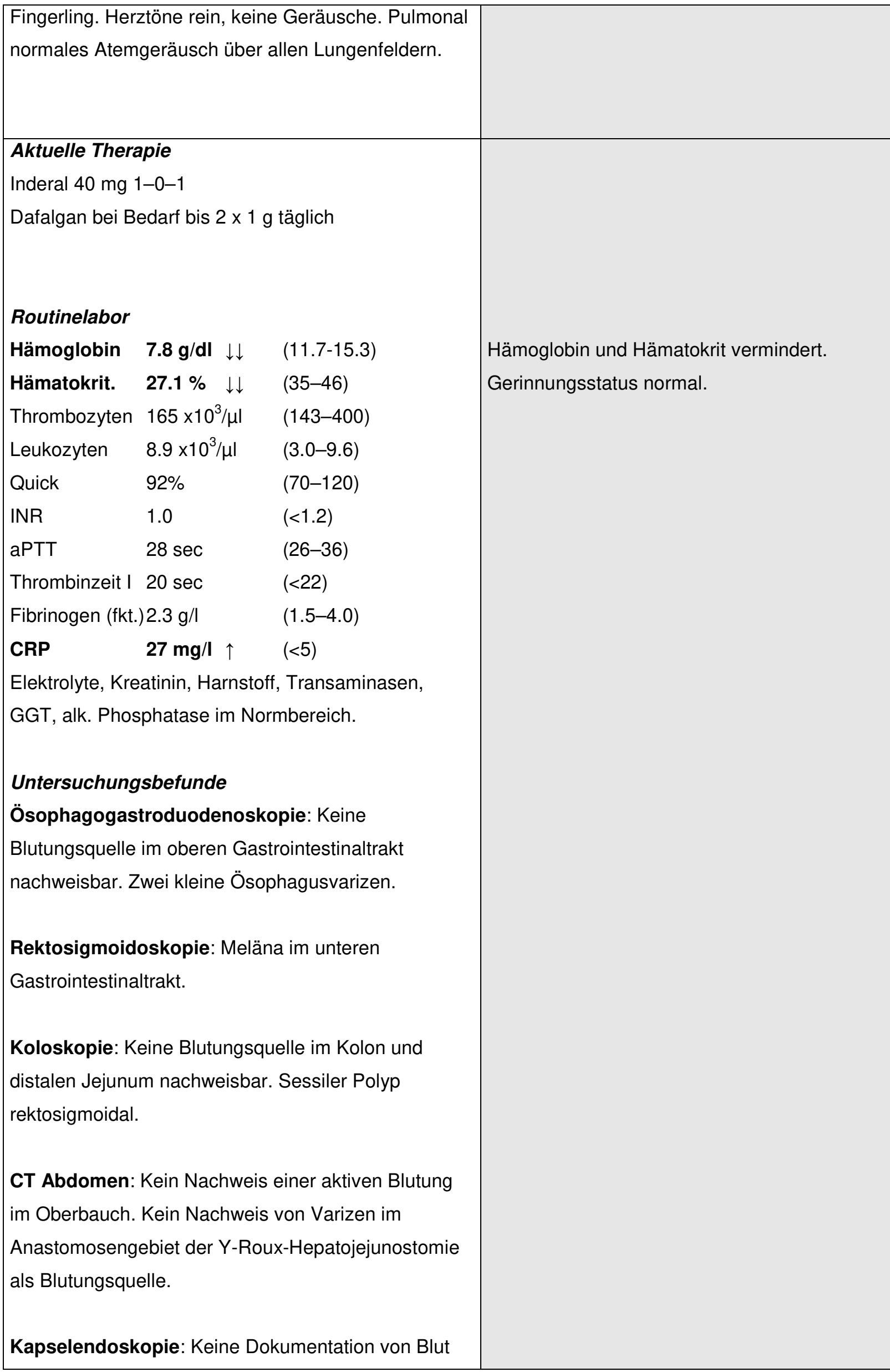




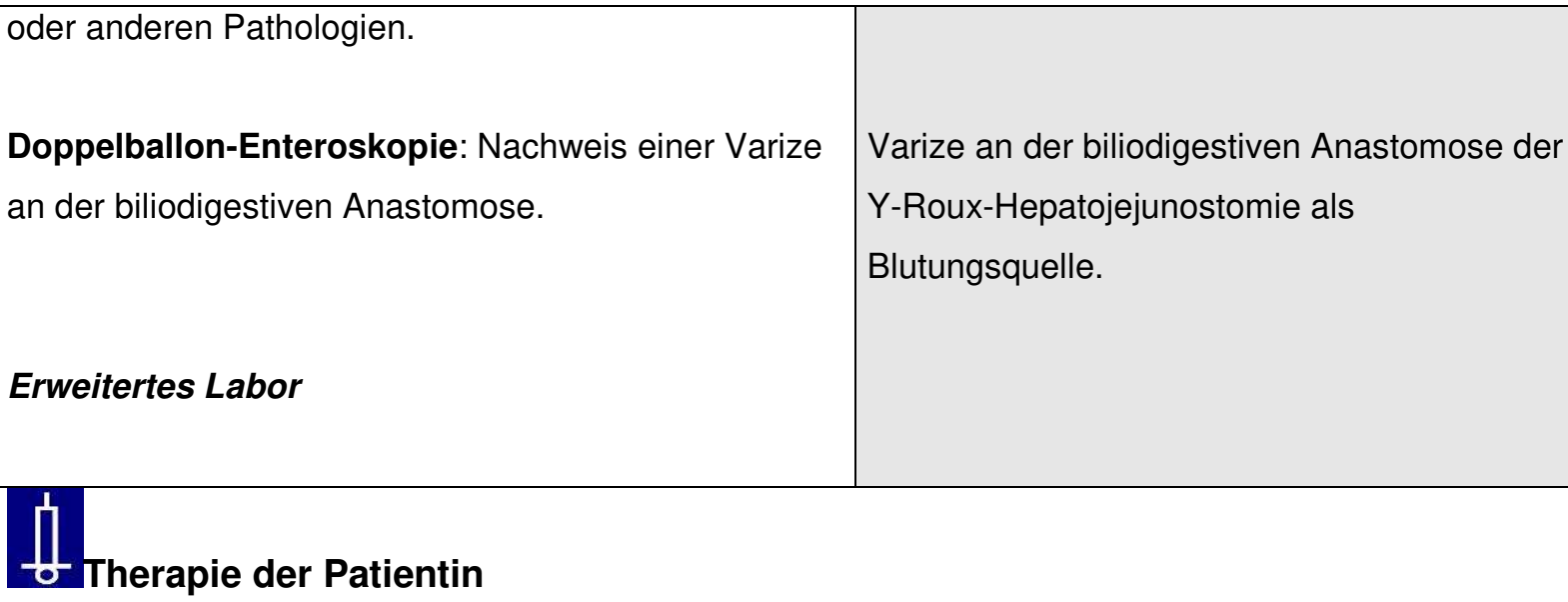

Erweitertes Labor

I.

Therapie der Patientin

- Hospitalisation bei akuter, anämisierender gastrointestinaler Blutung.

- Volumegabe und Gabe von zwei Erythrozytenkonzentraten.

- Bei Eintritt Verabreichung von Sandostatin $\AA$ und Nexium $\AA$.

- Histoacrylsklerosierung der Varize während der Doppelballon-Enteroskopie.

- Polypektomie rektosigmoidal elektiv im Verlauf.

\section{Autoren}

Klinik und Poliklinik für Innere Medizin ${ }^{1}$, Klinik für Gastroenterologie und Hepatologie ${ }^{2}$, Universitätsspital Zürich ${ }^{1}$ Dr. med. Sabine Haller, ${ }^{1}$ Dr. med. Pierre Krayenbühl, ${ }^{2}$ Dr. med. Christoph Gubler

\section{Korrespondenzadresse}

Dr. med. Sabine Haller

Klinik und Poliklinik für Innere Medizin

Universitätsspital Zürich

Rämistrasse 100

8091 Zürich

sabine.haller@usz.ch

\section{Bibliographie}

1. Kumar R, Mills AM. Gastrointestinal Bleeding. Emerg Med Clin North Am. 2011;29: 239-252.

2. Hussain H, Lapin S, Cappell MS. Clinical scoring systems for determining the prognosis of gastrointestinal bleeding. Gastroenterol Clin North Am. 2000;29: 445-464.

3. Wilcox CM, Clark WS. Causes and outcome of upper and lower gastrointestinal bleeding: the Grady Hospital experience. South Med J. 1999; 92: 44-50.

4. Longstreth GF. Epidemiology and outcome of patients hospitalized with acute lower gastrointestinal hemorrhage: a population-based study. Am J Gastroenterol. 1997; 92: 419-424. 
5. van Leerdam ME, Vreeburg EM, Rauws EA, et al. Acute upper GI bleeding. Did anything change? Time trend analysis of incidence and outcome of acute upper Gl bleeding between 1993/1994 and 2000. Am J Gastroenterol. 2003; 98: 1494-1499.

6. Masannat YA, Harron M, Harinath G. Nonsteroidal anti-inflammatory drugs-associated colopathy. ANZ J Surg. 2010; 80: 96-99.

7. Surveillance, Epidemiology, and End Results (SEER) Program, 1972-1992.

8. DiMaio CJ, Stevens PD. Nonvariceal upper gastrointestinal bleeding. Gastrointest Endoc Clin N Am. 2007; 17:253-272.

9. Lin S, Rockey DC. Obscure gastrointestinal bleeding. Gastroenterol Clin North Am. 2005; 34: 679-698.

10. Lewis BS. Obscure GI bleeding in the world of capsule endoscopy, push, and double balloon enteroscopies. Gastrointest Endosc. 2007; 66: S66-68.

11. Raju S, Gerson L, Das A, Lewis B. American Gastroenterological Association (AGA) Institute technical review on obscure gastrointestinal bleeding. Gastroenterology 2007; 133: 1697-1717..

12. Gupta PK, Fleischer DE. Nonvariceal upper gastrointestinal bleeding. Med Clin North Am. 1993; 77: $973-992$.

13. Huang CS, Lichtenstein DR. Nonvariceal upper gastrointestinal bleeding. Gastroenterol Clin North Am. 2003; 32: 1053-1078.

14. Barnert J, Messmann H. Diagnosis and management of lower gastrointestinal bleeding. Nat Rev Gastroenterol Hepatol. 2009; 6: 637-646.

15. Zuckerman GR, Prakash, C. Acute lower intestinal bleeding. Part II: etiology, therapy, and outcomes. Gastrointest Endosc. 1999; 49: 228-238.

16. American College of Physicians, Medical Knowledge Self-Assessment Program 15, Gastroenterology and Hepatology, 2011. 


\section{Fragen zu Blut ab ano}

\section{Frage 1}

Eine obskure Blutung kann folgende Merkmale aufweisen: (Mehrfachauswahl, 3 richtige Antworten)

a) Klinisch evidente Blutung mit sichtbarem Blut.

b) Blutung aus dem Gastrointestinaltrakt, deren Ursache nach Gastroskopie, Koloskopie und Dünndarmabklärung unklar bleibt.

c) Blutungsart, die nur im oberen GIB auftritt.

d) Blutungsquelle, die erst durch eine Kapselendoskopie identifiziert werden kann.

e) Präsentation der GIB durch einen positiven Fäkaltest und/oder durch eine Eisenmangelanämie, ohne klinisch sichtbaren Blutverlust.

\section{Frage 2}

Welches ist die häufigste Blutungsursache des oberen Gastrointestinaltrakts?

(Einfachauswahl, 1 richtige Antwort)
a) Angiodysplasie
b) Peptisches Ulkus
c) Mallory-Weiss-Läsion
d) Neoplasie
e) Erosion

\section{Frage 3}

Welche Untersuchung steht bei der Abklärung einer akuten Gastrointestinalblutung nicht im Vordergrund? (Einfachauswahl, 1 richtige Antwort)
a) Gastroduodenoskopie
b) Angiographie
c) Koloskopie
d) Abdomensonographie
e) Doppelballonenteroskopie

\section{Frage 4}

1. Eine Tc-99m-Pertechnetat-Szintigraphie ist bei geringem Blutverlust gegenüber einer Angiographie im Vorteil, 
weil

2. man damit eine Blutung bei einer niedrigen Blutungsrate ab $0,1 \mathrm{ml} / \mathrm{min}$ identifiziert kann.
a) 1. richtig, 2. falsch
b) 1. falsch, 2. richtig
c) alles richtig
d) 1. richtig, 2. richtig, Verknüpfung falsch
e) alles falsch

\section{Frage 5}

Welches ist der erste Schritt bei der Abklärung einer obskuren Gastrointestinalblutung?

(Einfachantwort, 1 richtige Antwort)
a) Angio-CT
b) Kapselendoskopie
c) Doppelballonenteroskopie
d) Laparoskopie
e) Second-look Endoskopien 


\section{Antworten zu den Fragen zu Blut ab ano aus PRAXIS Nr. 1}

\section{Frage 1}

Richtig sind Antworten a), b) und e).

Bei der obskuren Blutung handelt es sich um eine Blutung aus dem Gastrointestinal-Trakt, die anhaltend oder wiederholt auftritt, ohne eine identifizierte Blutungsquelle nach oberer und unterer Endoskopie und Abklärung des Dünndarms.

Sowohl eine overte (klinisch evidente Blutung mit sichtbarem Blut) als auch die okkulte (Präsentation der GIB durch einen positiven Fäkaltest und/oder durch eine Eisenmangelanämie, ohne klinisch sichtbaren Blutverlust) gastrointestinale Blutung können sich als obskure Blutung präsentieren. Eine obskure Blutung kann im gesamten Gastrointestinal-Trakt auftreten.

\section{Frage 2}

\section{Richtig ist Antwort b).}

Die häufigste Ursache einer oberen gastrointestinalen Blutung ist das peptische Ulkus, das $30-50 \%$ aller Fälle von oberen Gastrointestinal-Blutungen ausmacht und oft als Folge einer Helicobacter-pyloriInfektion oder von NSAR-Gebrauch auftritt. Die anderen aufgeführten Pathologien treten im Vergleich weniger häufig als Blutungsursachen auf.

\section{Frage 3}

\section{Richtig ist Antwort d).}

Die Abdomensonographie hat keinen Stellenwert bei der Abklärung einer akuten GastrointestinalBlutung. Die anderen aufgeführten diagnostischen Untersuchungen werden zur Suche einer Blutungsquelle bei einer akuten gastrointestinalen Blutung eingesetzt.

Zur Suche einer portalen Hypertonie ist die Abdomensonographie jedoch sehr wertvoll. Beim Vorliegen einer Splenomegalie, Aszites oder sicheren Zeichen einer Leberzirrhose wird die Vermutung einer Varizenblutung stark und der Abklärungsalgorithmus beeinflusst.

\section{Frage 4}

\section{Richtig ist Antwort c).}

Die Voraussetzung für eine erfolgreiche Suche einer Blutungsquelle mittels Angiographie ist ein Blutverlust von mindestens $1 \mathrm{ml} / \mathrm{min}$, das heisst drei bis vier Erythrozytenkonzentrate pro 24 Stunden. In der Tc-99m-Pertechnetat-Szintigraphie kann eine Blutung bei viel niedriger Blutungsrate (ab 
$0.1 \mathrm{ml} / \mathrm{min}$ ) identifiziert werden als in der Angiographie. Die Befunde einer Szintigraphie sind allerdings unspezifisch, eine Blutung kann nur einer generellen Region zugeordnet werden.

\section{Frage 5}

\section{Richtig ist Antwort e).}

Läsionen im Magen- oder Darmlumen können endoskopisch in bis zu 10\% verpasst werden, dies insbesondere in Notfallsituationen oder bei unzureichend vorbereitetem Kolon. Es ist daher bei obskurer Blutung angezeigt, die obere und/oder untere Endoskopie mindestens einmal zu wiederholen als sogenannte Second-look-Endoskopien.

Die weiteren aufgeführten Abklärungsmassnahmen werden in unterschiedlichen Abklärungsstadien relevant, wenn die Blutungsquelle trotz wiederholter Endoskopie nicht gefunden wurde. 